\title{
A New Yield Function of Isotropic Metals Included One to Six-order Plas- tic Tensors
}

\author{
Yang Feng ${ }^{1,2, *}$, Liu Jun ${ }^{3}$, Wan Shaohua ${ }^{4}$ and Wang Xiaoyan ${ }^{3}$
}

${ }^{1}$ College of Architectural and Civil Engineering Nanchang University, Nanchang 330031, China; ${ }^{2}$ School of Architectural and Civil Engineering East China Institute of Technology, Nanchang 330013, China; ${ }^{3}$ East China Institute of Technology, Nanchang 330013, China; ${ }^{4}$ The NO. 92866 in the Chinese People's Liberation Army, Qingdao, 266405, China

\begin{abstract}
The yield function is very important in establishing the plastic constitutive relation and analyzing the plastic deformation. Hence, expanded on the general yield function in its Taylor to six-order plastic tensors, this article gives a new yield function on isotropic metal. Based on independent properties of the hydrostatic pressure for metal yield, such as derivation character, mechanical property invariance of isotropic metal etc., these plastic tensors are analyzed as traceless, totally symmetric and objective. And the yield function for isotropic metals can be degenerated to the one for identical and different property of tension-compression yield. Finally, by means of the results of Lode test, it is proved that the new yield function is quite suitable for those metal materials having both the identical and different properties of tensioncompression yield. There are many merits of this yield function, such as it includes only 2 parameters for material, i.e. simple form and generality etc. And this yield function will lay a theoretical foundation for analyzing mechanical properties of metal materials.
\end{abstract}

Keywords: Hydrostatic, Isotropic metal, Plastic tensor, The yield function.

\section{INTRODUCTION}

The yield function is vital and necessary to describe the plastic deformation of metal. At present, there are hundreds of yield functions of metal, such as functions suggested by Hill, Hershey, Balart, Hosford, Man C.-S and Huang et al., to precisely describe the mechanical behavior of metals [12 ]. However, these yield functions are only suitable for anisotropic metals, but not on isotropic ones as the studies on their yield are so far very few. Some investigators reported that both Tresca and Von-mises yield criterion are usually used to establish the plastic constitutive relation and analyze the plastic deformation [3-5].

Based on the results of simple tension test for mild steel, Tresca yield function is shown in Eq. 1. However, this function is simple, since it doesn't consider the second principal stress. If the principal stresses are unknown, the expression of Tresca yield function maybe extremely complicated. Also, because of the corner point in the $\pi$ plane, the Tresca yield function is difficult to be dealt with during solving the equations [6].

$\max \left\{\left|\sigma_{1}-\sigma_{2}\right|,\left|\sigma_{2}-\sigma_{3}\right|,\left|\sigma_{3}-\sigma_{1}\right|\right\}=0$

Considering the second principal stress, Von-mises yield function, Eq. (2), is derived from the results of simple tension

*Address correspondence to this author at the School of College of Architectural and Civil Engineering Nanchang University, Nanchang, Jiangxi, 330013, PR. China; Tel: +86 0791 83879022; Fax: 0791 83879022;

E-mail: kittyyangfeng@126.com test and pure shear experiment. However, it is inadaptable for both the identical and different properties of tensioncompression yield, it doesn't consider the hydrostatic pressure of metals having no effect on yield [7].

$\left(\sigma_{1}-\sigma_{2}\right)^{2}+\left(\sigma_{2}-\sigma_{3}\right)^{2}+\left(\sigma_{3}-\sigma_{1}\right)^{2}=2 \sigma_{0}^{2}$

Microscopically metal is an aggregate of numerous tiny crystallites. Therefore, we can consider metal as isotropic materials in engineering. Because of the hydrostatic pressure of metals having no effect on yield and isotropic hypothesis, this article derives a new yield function of isotropic metal through expanding the traditional yield function by Taylor yield function and intercepting the six order. As well, the new yield function of isotropic metals can reduce to the one of the identical and different properties of tensioncompression yield. Finally, simple uniaxial tensioncompression experiment can be used to determine the parameters.

\section{ESTABLISHING THE NEW YIELD FUNCTION}

The yield function, $f(\sigma)$, is usually expressed by stress. When the metal is in the yield state, we have $f(\sigma)=0$. Conversely, the smooth yield function $f(\sigma) \neq 0$, as shown by Eq. 3, is expressed in its Taylor expansion at $\sigma=0$.

$$
\begin{aligned}
& f(\sigma)=f(\mathbf{0})+\frac{\partial f(\mathbf{0})}{\partial \sigma_{i j}}\left(\sigma_{i j}\right)+\frac{1}{2 !} \frac{\partial^{2} f(\mathbf{0})}{\partial \sigma_{i j} \partial \sigma_{k l}} \sigma_{i j} \sigma_{k l}+\frac{1}{3 !} \frac{\partial^{3} f(\mathbf{0})}{\partial \sigma_{i j} \partial \sigma_{k l} \partial \sigma_{p q}} \sigma_{i j} \sigma_{k l} \sigma_{p q} \\
& +\frac{1}{S !} \frac{\partial^{4} f(\mathbf{0})}{\partial \sigma_{i j} \partial \sigma_{k l} \partial \sigma_{p q} \partial \sigma_{r s}} \sigma_{i j} \sigma_{k l} \sigma_{p q} \sigma_{r s}+o(\|\sigma\|)=0
\end{aligned}
$$


Because of $f(\mathbf{0}) \neq 0$, we obtained the yield function by truncating the preceding expansion for each $S=1,2,3$. Then the Eq. (3) can be reduced to Eq. 4.

$$
-1+\mathbf{L}_{i j} \sigma_{i j}+\mathbf{M}_{i j k l} \sigma_{i j} \sigma_{k l}+\mathbf{T}_{i j k l p q} \sigma_{i j} \sigma_{k l} \sigma_{p q}=0
$$

Where, $\mathbf{L}_{i j}, \mathbf{M}_{i j k l}, \mathbf{T}_{i j k l p q}$ are respectively secondorder, four-order, and six-order plastic tensors of isotropic metals, as follows:

$$
\begin{aligned}
& \mathbf{L}_{i j}=\frac{-1}{f(\mathbf{0})} \frac{\partial f(\mathbf{0})}{\partial \sigma_{i j}} \\
& \mathbf{M}_{i j k l}=\frac{-1}{2 f(\mathbf{0})} \frac{\partial^{2} f(\mathbf{0})}{\partial \sigma_{i j} \partial \sigma_{k l}} \mathbf{T}_{i j k p q}=\frac{-1}{6 f(\mathbf{0})} \frac{\partial^{3} f(\mathbf{0})}{\partial \sigma_{i j} \partial \sigma_{k l} \partial \sigma_{p q}}
\end{aligned}
$$

\subsection{Conditions for the Yield Function of Isotropic Metals}

\section{(1) Total Symmetries}

Because of the reciprocal theorem of shear stress and Eq. 3, we can obtain Eq. 6 from the invariance principle on the exchange order of derivation [8].

$$
\begin{aligned}
& \mathbf{L}_{i j}=\mathbf{L}_{j i} \\
& \mathbf{M}_{i j k l}=\mathbf{M}_{k l i j}=\mathbf{M}_{j i k l} \\
& \mathbf{T}_{i j k l p q}=\mathbf{T}_{j i k l p q}=\mathbf{T}_{k l i j p q}=\mathbf{T}_{p q k l i j}
\end{aligned}
$$

\section{(2) Traceless}

The shear stress appeared by external force made the atoms slip, then the yield of metal is aroused from none recovery for the atoms slip during deformation. And the hydrostatic pressure has no effects on the shear stress [9]. Hence, the yield function of metal sheet must be satisfied with the Eq. 7 .

$$
f\left(\sigma_{i j}-m \delta_{i j}\right) \equiv f\left(\sigma_{i j}\right), \quad \forall m \in R
$$

$\delta_{i j}$ is Kronecker tensor; When $\forall i, j, k, l, p, q \in\{1,2,3\}$, based on the equality of the corresponding plastic tensor, the combination of Eqs. 3 and 6 lead to Eq.8.

$$
\mathbf{L}_{i i}=0 \quad \mathbf{M}_{i j k k}=0 \quad \mathbf{T}_{i j k p p}=0
$$

\section{(3) Objectivity}

The rotation of external force and object has no effect on the yield of isotropic metal, that is, when external force and object rotate, the yield function for isotropic metals must satisfy the objectivity [10].

Let $\mathrm{R}$ denote the rotation tensor, then the Euler angle will be $\psi, \theta, \phi$, we have [11]

$$
\begin{aligned}
& \mathrm{R}(\psi, \theta, \phi)=\left[\begin{array}{ccc}
\cos \psi \cos \theta \cos \phi-\sin \psi \sin \phi & -\cos \psi \cos \theta \sin \phi-\sin \psi \cos \phi & \sin \theta \\
\sin \psi \cos \theta \cos \phi+\cos \psi \sin \phi & -\sin \psi \cos \theta \sin \phi+\cos \psi \cos \phi & \sin \theta \\
-\sin \theta \cos \phi & \sin \theta \sin \phi & \cos \theta
\end{array}\right] \\
& S O_{3}=\{\mathbf{R}(\psi, \theta, \phi) \mid 0 \leq \psi \leq 2 \pi, 0 \leq \theta \leq \pi, 0 \leq \phi \leq 2 \pi\}
\end{aligned}
$$

Assumed that the external force and object rotate by R, the stress becomes:

$$
\sigma_{i j}(\mathbf{R})=R_{i p} R_{j q} \sigma_{p q}(\mathbf{I})
$$

Where there the stress is $\left(\sigma_{p q}(\mathbf{I})\right)$ before rotating, there is stress $\left(\sigma_{i j}(\mathbf{R})\right)$ after rotating.

Hence, the yield function must be met with Eq. 11 .

$$
f(\boldsymbol{\sigma}(\mathbf{I})) \equiv f(\boldsymbol{\sigma}(\mathbf{R}))=0, \quad \forall \mathbf{R} \in \mathbf{S O}(\mathbf{3})
$$

Put Eq. 3 into 11, based on the equality of the corresponding polynomial, the second-order, forth-order, and sixth-order plastic tensors respectively are shown by Eq.12.

$$
\begin{aligned}
& \mathbf{L}_{i j}(\mathbf{R})=R_{i p} R_{j q} \mathbf{L}_{p q}(\mathbf{I}) \\
& \mathbf{M}_{i j k l}(\mathbf{R})=R_{i p} R_{j q} R_{k r} R_{l s} \mathbf{M}_{p q r s}(\mathbf{I}) \\
& \mathbf{T}_{i j k l p q}(\mathbf{R})=R_{i a} R_{j b} R_{k c} R_{l d} R_{p e} R_{q f} \mathbf{T}_{a b c d e f}(\mathbf{I})
\end{aligned}
$$

Where, $\mathbf{L}_{i j}(I), \quad \mathbf{M}_{i j k l}(I)$, and $\mathbf{T}_{i j k p q}(I)$, are the plastic tensors of referred grains before rotating; $\mathbf{L}_{i j}(R)$, $\mathbf{M}_{i j k l}(R)$, and $\mathbf{T}_{i j k p q}(R)$, are the plastic tensors of referred grains after rotating.

\section{(4) Isotropy}

If the materials are isotropic, the yield must satisfy the condition that the material properties cannot change after rotation by $\mathrm{R}$, in $\forall \mathbf{R} \in \mathbf{S O}(\mathbf{3})$, that is, the plastic tensor for isotropic materials is shown as follows [12]:

$$
\begin{aligned}
& \mathbf{L}_{i j}(\mathbf{R})=\mathbf{L}_{i j}(\mathbf{I}) \\
& \mathbf{M}_{i j k l}(\mathbf{R})=\mathbf{M}_{i j k l}(\mathbf{I}) \\
& \mathbf{T}_{i j k l p q}(\mathbf{R})=\mathbf{T}_{i j k l p q}(\mathbf{I})
\end{aligned}
$$

Put Eq. 13 into 12, we can obtain Eq. 14.

$$
\begin{aligned}
& \mathbf{L}_{i j}(\mathbf{I})=R_{i p} R_{j q} \mathbf{L}_{p q}(\mathbf{I}) \\
& \mathbf{M}_{i j k l}(\mathbf{I})=R_{i p} R_{j q} R_{k r} R_{l s} \mathbf{M}_{p q r s}(\mathbf{I}) \\
& \mathbf{T}_{i j k p q}(I)=R_{i a} R_{j b} R_{k c} R_{l d} R_{p e} R_{q f} \mathbf{T}_{a b c d e f}(\mathbf{I})
\end{aligned}
$$

The isotropic materials plastic tensors can be obtained from the homogenization [13].

$$
\begin{aligned}
& \mathrm{L}(\mathrm{I})=\frac{1}{8 \pi^{2}} \int_{\mathrm{SO}_{3}} \mathrm{~L}(\mathrm{R}) d g \\
& \mathrm{M}(\mathrm{I})=\frac{1}{8 \pi^{2}} \int_{\mathrm{SO}_{3}} \mathrm{M}(\mathrm{R}) d g \\
& \mathrm{~T}(\mathrm{I})=\frac{1}{8 \pi^{2}} \int_{\mathrm{SO}_{3}} \mathrm{~T}(\mathrm{R}) d g
\end{aligned}
$$

Where, $\mathbf{S O}_{3}$ is a group of rotating tensors, and so $\mathbf{d} g=\sin \theta \mathbf{d} \psi \mathbf{d} \theta \mathbf{d} \varphi$.

\subsection{The General Form of the New Yield Function}

When the plastic tensors meet the conditions of Eqs. 5, 7, 11 and 13, they maybe become Eq. (16). 
$\mathbf{L}_{i j}=0$

$\mathbf{M}_{i j k l}=\chi B_{i j k l}^{(1)}-3 \chi B_{i j k l}^{(2)}$

$\mathbf{T}_{i j k l p q}=-2 \eta C_{i j k l p q}^{(1)}+9 \eta C_{i j k l p q}^{(2)}-9 \eta C_{i j k l p q}^{(3)}$

Where,

$B_{i j k l}^{(1)}=\delta_{i j} \delta_{k l}$

$B_{i j k l}^{(2)}=\frac{1}{2}\left(\delta_{i k} \delta_{j l}+\delta_{i l} \delta_{j k}\right)$

$C_{i j k l p q}^{(1)}=\delta_{i j} \delta_{k l} \delta_{p q}$

$C_{i j k l p q}^{(2)}=\frac{1}{3}\left(\delta_{i j} B_{k l p q}^{(2)}+\delta_{k l} B_{i j p q}^{(2)}+\delta_{p q} B_{i j k l}^{(2)}\right)$

$C_{i j k p q}^{(3)}=\frac{1}{4}\left(\delta_{i q} B_{j p k l}^{(2)}+\delta_{j q} B_{i p k l}^{(2)}+\delta_{i p} B_{j q k l}^{(2)}+\delta_{j p} B_{i q k l}^{(2)}\right)$

Combine Eq. 16 with 3, the new yield function on isotropic metal is shown as follows:

$$
\begin{aligned}
& f_{1}(\sigma)=M\left[\left(\sigma_{11}-\sigma_{22}\right)^{2}+\left(\sigma_{22}-\sigma_{33}\right)^{2}+\left(\sigma_{33}-\sigma_{11}\right)^{2}\right. \\
& \left.+6\left(\sigma_{12}^{2}+\sigma_{23}^{2}+\sigma_{31}^{2}\right)\right]+P\left[\left(2 \sigma_{11}-\sigma_{22}-\sigma_{33}\right)\right. \\
& \left(2 \sigma_{22}-\sigma_{11}-\sigma_{33}\right)\left(2 \sigma_{33}-\sigma_{11}-\sigma_{22}\right)+54 \sigma_{12} \sigma_{13} \sigma_{23}+ \\
& 9 \sigma_{12}^{2}\left(\sigma_{11}+\sigma_{22}-2 \sigma_{33}\right)+9 \sigma_{13}^{2}\left(\sigma_{11}+\sigma_{22}-2 \sigma_{22}\right)+ \\
& \left.9 \sigma_{23}^{2}\left(\sigma_{22}+\sigma_{33}-2 \sigma_{11}\right)\right]-1=0
\end{aligned}
$$

If we take $M=-\chi, \quad P=-\eta$, where $\chi$ and $\eta$ are material parameters, measured by experiment.

From Eq.17, the stresses of a quadratic and a cubic term are included in this new function, and it has only 2 material parameters, i.e. $\chi$ and $\eta$, which can be measured by some experiments.

So the form of the new yield function is not only simple but also has much higher theory and application value in engineering.

\section{DISCUSSION ABOUT THE DEGRADATION FORM OF YIELD FUNCTION}

Instead of the principle stress, the new yield function of isotropic metal materials is the Eq. 18.

$$
\begin{aligned}
& f_{2}(\boldsymbol{\sigma})=M\left[\left(\sigma_{1}-\sigma_{2}\right)^{2}+\left(\sigma_{2}-\sigma_{3}\right)^{2}+\left(\sigma_{3}-\sigma_{1}\right)^{2}\right]+P \\
& \left(2 \sigma_{1}-\sigma_{2}-\sigma_{3}\right)\left(2 \sigma_{2}-\sigma_{1}-\sigma_{3}\right)\left(2 \sigma_{3}-\sigma_{1}-\sigma_{2}\right)-1=0
\end{aligned}
$$

For metal materials of the identical property on tensioncompression yield, the yield strength of tension is $\sigma_{s}$, and the one of compression is $\sigma_{c}=\sigma_{s}$. On the contrary, for metal materials of different property for tension-compression yield, the yield strengths of tension-compression are respectively $\sigma_{c}$ and $\sigma_{s}$. So the principal stress states for uniaxial tensile and uniaxial compression tests are shown in (Table 1).

According to the kinds of state of the principle stress, for metal materials having both identical and different properties of tension-compression yield, the Eq. 18 respectively turns into Eq. 19 and 20.

$$
\begin{aligned}
& 2 M \sigma_{S}^{2}+2 P \sigma_{S}^{3}-1=0 \\
& 2 M \sigma_{S}^{2}-2 P \sigma_{S}^{3}-1=0 \\
& 2 M \sigma_{S}^{2}+2 P \sigma_{S}^{3}-1=0 \\
& 2 M \sigma_{c}^{2}-2 P \sigma_{c}^{3}-1=0
\end{aligned}
$$

By solving Eq. 19 and 20, we can obtain $\mathrm{M}$ and $\mathrm{P}$ for identical and different properties of tension-compression yield respectively, as follows in Eq. 21 and 22.

$$
\begin{aligned}
& M=\frac{1}{2 \sigma_{s}^{2}} \quad P=0 \\
& M=-\frac{\sigma_{s}-\sigma_{c}}{2 \sigma_{s}^{2} \sigma_{c}^{2}} \quad P=\frac{\sigma_{s}^{2}+\sigma_{c}^{2}-\sigma_{s} \sigma_{c}}{2 \sigma_{s}^{2} \sigma_{c}^{2}}
\end{aligned}
$$

In a word, firstly, the tension and compression yield strengths, i.e. $\sigma_{S}$ and $\sigma_{C}$, are measured by uniaxial tensile and uniaxial compression tests; secondly, from Eqs. 20 and 21 , the material parameters, $\mathrm{M}$ and $\mathrm{P}$, are determined by $\sigma_{S}$ and $\sigma_{C}$ respectively; finally, we can get the new yield function included One to Six-order Plastic Tensors by Eq. 18. Therefore, the 2 material parameters from the new yield function can also be identified only by uniaxial tensile and uniaxial compression tests.

\section{EXPERIMENTAL VERIFICATION}

In 1926, W. Lode proposed the Lode parameters, that is $\mu_{\sigma}, \varsigma$ and $\kappa$, by yield test in which the axial force and internal pressure was applied to the thin-wall cylinder made of mild steel $[14,15]$. And the formulas of $\mu_{\sigma}, \varsigma$ and $\kappa$ are given by Eq. 22 .

$$
\begin{aligned}
& \mu_{\sigma}=\frac{2 \sigma_{2}-\sigma_{1}-\sigma_{3}}{\sigma_{1}-\sigma_{3}} \\
& \varsigma=\frac{\sigma_{1}-\sigma_{3}}{\sigma_{s}} \\
& \kappa=\frac{\tau_{s}}{\sigma_{s}}
\end{aligned}
$$

Based on the results of $\mathrm{W}$. Lode experiments, the relation between $\varsigma$ and $\mu_{\sigma}$ has been analyzed by the yield criteria of Teresa, Von-mises and the new yield criterion of isotropic metals proposed in this thesis. And the results are shown in Fig. (1).

From Fig. (1), according to Tresca yield function, $\kappa$ must be equal to 0.5 , that is $\zeta$ is equal to 1 ; meanwhile, as for Von-mises yield function, $\kappa$ must be equal to

$$
\frac{\sqrt{3}}{3} \text {, that is } \zeta \text { is equal to } \frac{2}{\sqrt{3+\mu_{\sigma}^{2}}} \text {. }
$$


Table 1. The stress state of metals having both identical and different properties of tension-compression yield uniaxial tension and uniaxial compression tests.

\begin{tabular}{|c|c|c|c|c|}
\hline \multirow{2}{*}{\begin{tabular}{c}
\multirow{2}{*}{$\begin{array}{c}\text { Stress } \\
\text { state }\end{array}$} \\
\cline { 2 - 5 }
\end{tabular}} & \multicolumn{2}{|c|}{ identical property for tension-compression yield } & \multicolumn{2}{c|}{ different property for tension-compression yield } \\
\hline \hline$\sigma_{c}$ & $\sigma_{s}$ & uniaxial compression & uniaxial tension & uniaxial compression \\
\hline$\sigma_{1}$ & 0 & 0 & $\tau_{s}$ & 0 \\
\hline$\sigma_{3}$ & 0 & 0 & 0 & 0 \\
\hline
\end{tabular}

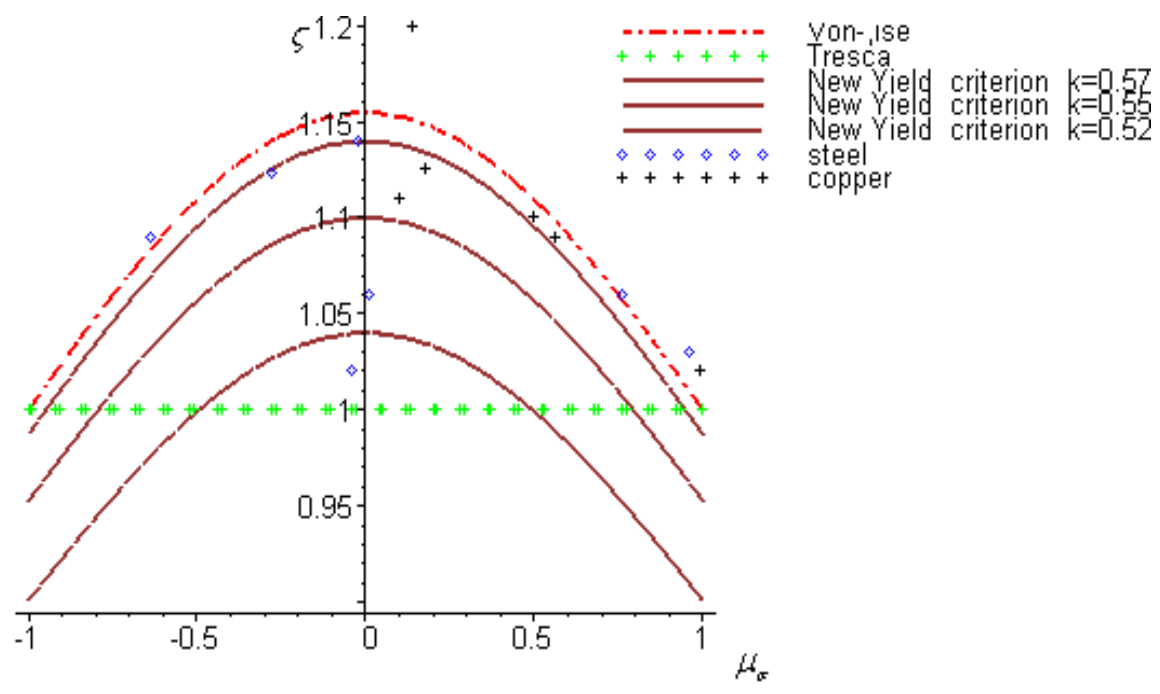

Fig. (1). The relation between $\zeta$ and $\mu_{\sigma}$ on kinds of yield function.

However, different materials have different value for $\kappa$. Because Tresca and Von-mises yield functions demand that $\kappa$ should be the determined value in practical engineering, the two yield function cannot be applied to the engineering. But when taking $\kappa$ to be a different value, the new yield function considering the six-order plastic tensors of the isotropic metals overcomes the shortcomings. Put Lode parameters into the new yield function, $\zeta$ gives Eq. 23.

$\varsigma=\frac{\sigma_{1}-\sigma_{3}}{\sigma_{s}}=\frac{2 \sqrt{3} \kappa}{\sqrt{3+\mu_{\sigma}^{2}}}$

According to Eq. 23, when

$\kappa=\frac{\sqrt{3}}{3}$,

the new yield function can be degenerated to Von-mises yield function.

The Fig. (1) shows that this new yield function is quite suitable for those metal materials, which had both identical and different properties of tension-compression yield. And there are only 2 material parameters, determined by uniaxial tensile and compression tests. It is both simple and more accurate than Tresca and Von-mises yield functions on describing the plastic deformity.

\section{CONCLUSION}

Based on the stress relation, the general form of a new yield function included the various degree terms for one from three on the isotropic metals and corresponding sixorder plastic tensor was deduced in this thesis;

A new yield function of the isotropic metals is derived from the isotropic plastic tensor based on the total symmetries, traceless, objectivity and isotropy. And there are many advantages, such as simple form and higher value in engineering;

The new yield function of isotropic metal transformed into Eq. 17, included the principle stress. And according to the metal materials, which have both the identical and different properties of tension-compression yield, the 2 material parameters are determined by uniaxial tensile and compression tests;

According to the results of Lode experiments, $\varsigma$ and $\mu_{\sigma}$ are calculated by the new yield function, and Tresca and Von- Mises yield functions. This new yield function has advantages of its simple form and is more suitable for describing both the yield and the plastic deformation of isotropic metals.

\section{CONFLICT OF INTEREST}

The authors confirm that this article content has no conflict of interest. 


\section{ACKNOWLEDGEMENTS}

This work was financially supported by the National Natural Science Foundation of China (51304050, 10972098, $11172122,51268043,61463005)$ and the Education Department of Jiangxi province science and technology research projects (GJJ13445, GJJ13446, GJJ150575); Natural Science Foundation of Jiangxi Province (20161BAB216142).

\section{REFERENCES}

[1] Niu Jie,Liu Guanglian Tian Jie. Comparsion of yield strength the ories wiyh experimental results [J]. Engeering Mechanics, 2014; 31(1): 181-187.

[2] Dorel Banabic. Sheet Metal Forming Processes [M]. SpringerVerlag Berlin and Heidelberg GmbH \& Co. K, 2008; 39-43.

[3] Yang Qiang, Chen Xin, Zhou Weiyuan. An anisotropic yield criterion based on the two-order fabric tensior [J] 2005; 22(6): 15-20.

[4] Yu Mao-hong, M. Yoshimine, Qiang Hong-fu ect. Advances and prospects for strength theory [J]. Engineering Mechanics, 2004; 21(6): $1-20$

[5] Mojia Huang, Chi-Sing Man.A Generalized Hostord Yield Function for Weakly-textured Sheets of Cubic Metals. [J] International J Plasticity, 2013; 41: 97-123.

[6] Zhao Dewen, Liu Xianghua, Wang Guodong. Yield criterion Based on the Mean Function of Tresca and Twin Shear Stress Yield Func- tion [J]. Journal of Northeastern University (Natural Science), 2002; 23(10): 976-979.

[7] Zheng Shu. The comparison for two yield function. [J]. Journal of the mechanics and practices, 2003; 25(2): 62-63.

[8] Mojia Huang, Chi-Sing Man.a generalized hostord yield function for weakly-textured sheets of cubic metals. [J] International J Plasticity, 2013; 41: 97-123.

[9] Wan Shaohua. On the constitutive relations of orthorhombic aggregates of cubic crystallites with effect the stress-strain's of highorder effects [M]. doctor Thesis, Nanchang University, 2013.

[10] Man C.-S., Huang M., Identification of material parameters in yield functions and flow rules for weakly textured sheets of cubic metals[J], International Journal of non-linear mechanics, 2001; 36: 501-514.

[11] Wu Ping. The Elastic-plastic Constitute Relation Connected with Texture Coefficients And Its Ultrasonic Measurement [M]. doctor Thesis, Nanchang University, 2013.

[12] Huang Mojia, Fu Mingfu .Prediction of Yield Functions on BBC Pycrystals[J]. Acta Mechanica Solida Sinica, 2006, 19(1): 75-85.

[13] Mojia Huang, Huahu Cheng. Determination of all elastic and plastic parameter for sheets of cubic metals only by uniaxial tension tests [J].European Journal of Mechanics A/Solid 2014; 10: 539547.

[14] Lode W. Versuche ber den einfluss dermittleren haupt spannung auf das fliessen der matalle eisen ,kupfer und nickel. Zeitsch Phys, 1926; 36: 913.

[15] Huang M.Model Verification of Lode's test results and yield function of isotropic FCC polycrystal,Acta Mechanica. 2011.

(C) Feng et al.; Licensee Bentham Open.

This is an open access articles licensed under the terms of the Creative Commons Attribution-Non-Commercial 4.0 International Public License (CC BY-NC 4.0) (https://creativecommons.org/licenses/by-nc/4.0/legalcode), which permits unrestricted, non-commercial use, distribution and reproduction in any medium, provided that the work is properly cited. 